\title{
A Novel and Simplest Green Synthesis Method of Reduced Graphene Oxide Using Methanol Extracted Vernonia Amygdalina: Large-Scale Production
}

\author{
Bayisa Meka Chufa $\mathbb{D}^{1},{ }^{1}$ Bedasa Abdisa Gonfa ${ }^{\mathbb{D}},{ }^{1}$ Teketel Yohannes Anshebo, ${ }^{2}$ \\ and Getachew Adam Workneh ${ }^{3}$ \\ ${ }^{1}$ Department of Applied Chemistry, School of Applied Natural Science, Adama Science and Technology University, P.O. Box 1888, \\ Adama, Ethiopia \\ ${ }^{2}$ Department of Chemistry, School of Natural Science, Addis Ababa University, P.O. Box 1176, Addis Ababa, Ethiopia \\ ${ }^{3}$ Department of Industrial Chemistry, College of Applied Science, Addis Ababa Science and Technology University, \\ P.O. Box 16417, Addis Ababa, Ethiopia
}

Correspondence should be addressed to Bayisa Meka Chufa; nathan.meka@astu.edu.et

Received 16 December 2020; Accepted 5 February 2021; Published 16 February 2021

Academic Editor: Da-Ren Hang

Copyright (c) 2021 Bayisa Meka Chufa et al. This is an open access article distributed under the Creative Commons Attribution License, which permits unrestricted use, distribution, and reproduction in any medium, provided the original work is properly cited.

\begin{abstract}
The large-scale production of high-quality graphene is the major focus of scientists and engineers recently. However, its massive manufacturing routes from its precursor graphene oxide (GO) are involved in the production of toxic gasses and consist of hazardous explosive steps that severely hurt and threaten ecological balance and human health. Therefore, in this study, we investigated the green, effective, and economical approach for the synthesis of graphene by using Vernonia amygdalina (VA) plant leaf extracts for the effective and efficient reduction of GO. The nonexplosive two-step synthesis of GO in a short period of time in the absence of an ice bath was used in this study. The appropriate solvent for the extraction of VA for the green synthesis of graphene was methanol, and the reducing and capping agent in the plant extract was identified to be terpenoids and polyphenols. The graphene/rGO obtained this way was characterized by UV-VIS, XRD, FTIR, SEM, HR-TEM, and EDAX that confirmed the successful reduction of GO to graphene under the hydrothermal process. The HR-TEM images showed the development of few layers of graphene. The FTIR result also shows the complete reduction of GO. Hence, methanol extracted VA leaves consisted of the most appropriate compounds for reducing and capping agent in the green synthesis and could be the preferred method for the large-scale production of graphene-based materials.
\end{abstract}

\section{Introduction}

Graphene, a two-dimensional, only one atom thick, and densely packed crystalline material, is the thinnest, lightest, and strongest material on Earth and the best conductor of heat and electricity known to man [1-5]. Recent reports have also mentioned that graphene materials are the ideal materials for energy storage devices and for the application of photocatalytic activities [6-8]. This material has been identified and analyzed by mechanical exfoliation of pyrolytic graphite a decade ago by Manchester university physicists Geim and Novoselov, because of which they won the noble prize in 2010 for their record-breaking work $[9,10]$. Even if the above method and epitaxial chemical vapor depositions are more preferable to produce precise pristine graphene, they are less effective for large-scale manufacturing $[11,12]$. The interest of scientists to investigate this material began 70 years ago, when Wallace explained some of the physical properties of graphite through the band theory of solids [13]. The carbon atoms are connected in a hexagonal honeycomb lattice in graphene and can be seen with the naked eye because it absorbs about $2.3 \%$ of light [14]. Recent studies showed that graphene is not only the strongest material ever measured but also the 
stiffest, and its current density is a million times that of copper [15]. This new material has shown a number of unique properties so that it is much more than just a scientific interest; it boasts a growing list of real-world applications [16-18].

Hence, the large-scale production method of graphene and its family should be pronounced by researchers. The most popular approach to produce large-scale graphene to date is the use of a strong oxidizing agent to obtain graphene oxide (GO) $[19,20]$. GO is the oxidized analogy of graphene from which we can only synthesize graphene on a large scale [21]. It is a nonstoichiometric chemical compound of carbon, hydrogen, and oxygen in variable ratios which largely depend on the processing methodologies [22-24]. GO possesses a large amount of oxygen functional groups that are presented to the carbon skeleton in the form of epoxide and hydroxyl across the basal plane (hydroxyl, carbonyl, and carboxyl) at the edge during chemical oxidation from the oxidizing agent [25-27]. This deliberate insertion of an oxygen-containing functional group into graphite is reflected by the increment of interlayer distance from $0.34 \mathrm{~nm}$ in graphite to $0.63 \mathrm{~nm}$ in GO [28]. Then, this product, firstly graphite oxide, and later on graphene oxide (after exfoliation by the addition of distilled water and ultrasonication) reveal the following special properties such as easy functionalization, hydrophilicity, and ability to convert to graphene (for large-scale production) and prevent the restoration of Van der Waals interaction between graphene layer in graphite leading to exfoliation upon sonication [26-30]. Therefore, the chemical exfoliation method is the most popular method to prepare GO, reduced graphene oxide (rGO), and graphene. GO is highly polar and hydrophilic but is a nonconductor of electricity because of the presence of a large amount of oxygen at its edge and basal plane [31, 32].

In this study, graphene oxide was synthesized by the novel modification of the improved method which was later on used as a precursor for the synthesis of reduced graphene oxide by the simplest green method. Solvent extracted compounds of Vernonia amygdalina (VA) plant were used to synthesize rGO. This method is cheap, where the reducing agent is abundantly locally available, and ecofriendly where it does not discharge any gas and toxic substances into the environment unlike the antioxidant chemicals such as hydrazine hydrate and ascorbic acid, and furthermore, the synthesis method is easy and scalable. In our preceding study, we identified the optimum condition and effective solvent extraction method to be used to extract compounds from VA for the synthesis rGO.

Hence, the objective of this study was to synthesis reduced graphene oxide using abundantly available plant extracts of Vernonia amygdalina as a reducing and capping agent. The objective also includes the large-scale synthesis of this recently significant attention captured graphene-based material at the nanoscale.

\section{Materials and Methods}

2.1. Materials. Graphite powder pract of 100 microns (99.5\% purity) from S D Fine Chem, methanol extracted leaf of Vernonia amygdalina, sulfuric acid (98\%), phosphoric acid $(75 \%)$, hydrogen peroxide (35\%), hydrochloric acid (37\%), potassium permanganate $(99.5 \%)$, barium chloride (99\%), methanol (99.8\%), and deionized water were purchased from a market and used directly without further treatment.

2.2. Synthesis of Graphene Oxide (GO). GO was synthesized by making a slight modification to the improved method [33]. A mixture of $\mathrm{H}_{2} \mathrm{SO}_{4} / \mathrm{H}_{3} \mathrm{PO}_{4}$ in a volume ratio of $9: 1$ was prepared. A second mixture of graphite $/ \mathrm{KMnO}_{4}$ in a mass ratio of $1: 6$ was prepared. The $\mathrm{H}_{2} \mathrm{SO}_{4} / \mathrm{H}_{3} \mathrm{PO}_{4}$ acid mixture was poured into the beaker containing graphite$\mathrm{KMnO}_{4}$ mixture slowly at room temperature while continuous stirring. A deep green color solution was formed due to the production of highly oxidizing dimanganese heptoxide $\mathrm{Mn}_{2} \mathrm{O}_{7}$. Under controlled temperature of water bath at $80^{\circ} \mathrm{C}$, the solution was heated while stirring using a magnetic stirrer for six hours. The hot and slightly brown solution was taken off the water bath and added to $400 \mathrm{ml}$ distilled ice water to terminate the reaction. Then, $5 \mathrm{ml}$ of $\mathrm{H}_{2} \mathrm{O}_{2}$ was added dropwise because of which a yellowish colored solution was formed. The purpose of the addition of $\mathrm{H}_{2} \mathrm{O}_{2}$ was to stop the oxidation reaction by directly reacting with the excess of potassium permanganate. The reaction product was washed with 1 liter of $5 \% \mathrm{HCl}$ aqueous solution and distilled water four times each until the $\mathrm{pH}_{\mathrm{pzc}}$ was obtained. The complete removal of $\mathrm{SO}_{4}{ }^{-2}$ ion from the solution was checked by the addition of $\mathrm{BaCl}_{2}$ to the supernatant. Its presence produces a white precipitate of $\mathrm{BaSO}_{4}$. The resulting paste was dried in an oven at $60^{\circ} \mathrm{C}$ for 8 hours to obtain the brown graphene oxide sample.

\subsection{Synthesis of Reduced Graphene Oxide (rGO).} Graphene oxide was deoxygenated using Vernonia amygdalina (locally called "dhebicha") plant extract to produce rGO. $10 \mathrm{ml}$ of methanol extracted compounds of bitter leaf (dhebicha) was added to $100 \mathrm{ml}$ of the brown solution of graphene oxide. The mixture was heated with a heater while stirring using a magnetic stirrer at $70^{\circ} \mathrm{C}$ for an hour. At this temperature, the solvent was evaporated leaving the reducing and capping agent behind. The black paste was then taken off and cooled at room temperature after which excess $\mathrm{H}_{2} \mathrm{O}_{2}$ was added to separate the solvent from the rGO. The clear solvent and the black residue (rGO) were separated easily by Whatman number 1 filter paper. The black and foam-like structure was washed multiple times with distilled water and dried in an oven at $50^{\circ} \mathrm{C}$ for 5 hours. After drying, the sample was ground finely using glass mortar and pestle and stored for further use.

2.4. Characterization Techniques. The presence of oxygencontaining functional groups on the basal plane or edge of the carbon framework in GO and rGO was determined by Fourier-transform infrared spectroscopy, FTIR (Perkin Elmer, USA). GO and rGO pellets were prepared using $\mathrm{KBr}$ as a mulling agent, and the sample was analyzed in the range 
between $400 \mathrm{~cm}^{-1}$ and $4000 \mathrm{~cm}^{-1}$. The morphology of the samples was analyzed using scanning electron microscopy, SEM (Jeol, JSM 6460LV). X-ray diffraction, XRD of graphite, GO, and rGO were recorded on a Panalytical PW 3040 X' pert MPD X-ray diffractometer with $\mathrm{Cu}-\mathrm{K} \alpha \mathrm{X}$-ray radiation sources at $40 \mathrm{keV}$ and $30 \mathrm{~mA}$. XRD spectra were recorded to determine the purity and crystallinity of the samples. Highresolution transmission electron microscopy, HR-TEM (Philips CM-1000 with a tungsten filament), coupled with EDAX was used to determine the morphology, measure the size of the particles, and quantify the elemental composition. Optical absorption properties of graphite $\mathrm{GO}$ and $\mathrm{rGO}$ were examined by ultraviolet-visible spectroscopy, UV-Vis (SM, UV-1600 Maadab-India). Energy-dispersive X-ray analysis (EDX), referred to as EDS or EDAX, was used in this work to identify the elemental composition of (GO, rGO).

\section{Result and Discussion}

3.1. Visual Observation. As shown in Figures 1(a)-1(d), four main different colors were observed during the stepwise synthesis of rGO. The mixing of the two mixtures (a mixture of graphite/ $\mathrm{KMnO}_{4}$ and $\mathrm{H}_{2} \mathrm{SO}_{4} / \mathrm{H}_{3} \mathrm{PO}_{4}$ ) resulted in the formation of a dark green colored thick solution as shown in Figure 1(a). This color was due to the formation of the high oxidizing agent dimanganese heptoxide $\left(\mathrm{Mn}_{2} \mathrm{O}_{7}\right)$ [31, 34] from the reaction of $\mathrm{MnO}_{3}^{+}$and $\mathrm{MnO}_{4}^{-}$, and in the meanwhile, the temperature of the solution was increased to $45^{\circ} \mathrm{C}$. The thick slurry solution observed in Figure 1(b) was due to prolonged reaction under heating and stirring. The presence of unreacted potassium peroxide was observed by the formation of yellowish color by the addition of $\mathrm{H}_{2} \mathrm{O}_{2}$ which disappeared later by intensive washing. The brown color as indicated in Figure 1(c) was obtained which is one of the confirmations of the formation of graphene oxide nanostructure. The dark black suspension formed as shown in Figure 1(d) is the sign of GO reduction to produce rGO. The images of powders depicted in Figures 2(a) and 2(b) are GO and rGO, respectively. The brown powder on the lefthand side (a) is GO, and the black powder on the right-hand side (b) is the reduced form of GO called rGO.

The reduction was done by using Vernonia amygdalina plant extract, and this result confirmed that bitter leaf extract is an eco-friendly and excellent reducing agent for the nanosynthesis of rGO.

3.2. Principles of $\mathrm{GO}$ Reduction. $\mathrm{KMnO}_{4}$ donates a large amount of oxygen to the precursor graphite to produce GO in the presence of chopping and intercalating agent $\mathrm{H}_{2} \mathrm{SO}_{4}$ / $\mathrm{H}_{3} \mathrm{PO}_{4}$. Hence, GO possesses a large amount of oxygencontaining functional group at its basal plane (epoxy and hydroxyl) and at its edge (carboxyl, carbonyl, and hydroxyl). GO makes use of these active sites to react with the other active sites on the alkaloids, polyphenols, and carboxyl of the Vernonia amygdalina extract. In this study, the hydroxyl of methanol extracted polyphenols were reacted with the hydroxyl and epoxy group on the GO favoring a ring formation which was followed by ring opening which facilitates the reduction of GO. Besides, the hydroxyl group on the polyphenols of plant extract was reacted with the carboxylic group of the GO generating an ester in the condensation process. In each step of this process, a cleavage of bonds occurred, and the reduction of GO progressed. In the meanwhile, the longchain plant extracted compounds such as the carbonyl, hydroxyl, and esters were involved in the capping agent of the synthesis through $\pi-\pi *$ stacking interaction principle rGO [25]. The nucleophilic substitution reactions happened simultaneously as in the case of the $\mathrm{SN}_{2}$ principle [31].

3.3. UV-Vis Absorption Analysis. The existence of conjugate bonds, the extent to which it exists, and the degree of redox reaction that has taken place in GO and rGO were monitored by UV-Vis spectroscopy measurements. The presence of various extents of conjugation in GO and rGO is determined from the $\lambda_{\text {max }}$ value of the UV-Vis spectra. UV-Vis spectroscopy generated independent $\lambda_{\text {max }}$ values for GO and rGO with the larger value corresponding to rGO spectra as shown in Figure 3.

As shown in Figure 3, the $\pi-\pi *$ transition $\lambda_{\max }$ values for GO are observed at $230 \mathrm{~nm}$ and $\lambda_{\text {max }}$ for rGO was at $259 \mathrm{~nm}$ which means that a peak at a larger wavelength $(259 \mathrm{~nm})$ was recorded for rGO. This specifies that most oxygen functionalities were removed from the surface of $\mathrm{GO}$ and conjugations $\mathrm{C}=\mathrm{C}$ remain restored. This indicates that there are a large number of $-\mathrm{C}=\mathrm{C}$ - free conjugations in $\mathrm{rGO}$. In this case, a small amount of energy is required to remove its electron. As the absorption peak decreases, there must be a decrease in the delocalization of electrons which means on the other hand a decrease in the degree of conjugation. In this case, a large amount of energy that is required to remove electrons is needed because of their strong attachment to the parent in the localized bond. Here, a more electronegative oxygen atom is occupied either at the basal plane or at the edge so that less delocalization effect was observed. A shoulder peak was also observed at $300 \mathrm{~nm}$ as shown in Figure 3 which was attributed to $n-\pi *$ transition. The $n-\pi *$ transition is a transition that occurred from either hydroxyl or carboxyl or carbonyl group in the structure of GO and rGO. As a result of the reduction reaction, GO is deoxygenated and converted into rGO with a less number of attached oxygen, and there must be a greater number of $n$ $\pi *$ transition in GO than rGO. The higher the intensity of $\mathrm{n}-\pi *$ transition, the higher the degree of oxidation.

3.4. X-Ray Diffraction (XRD). The changes in the crystal structure of graphite followed by its oxidation to produce GO and subsequent reduction to produce rGO were evaluated by XRD patterns as shown in Figure 4. Besides structural arrangement, this characterization technique was used to monitor the extent of oxidation and purity of the material under examination. The interlayer spacing and its corresponding sharp peak at the $2 \theta$ value of graphite are $0.34 \mathrm{~nm}$ and $26.4^{\circ}$, respectively, which is in good agreement with a graphitic nature and exactly matches the literature values $[35,36]$. Regarding GO, the $2 \theta$ value was shifted to $10^{\circ}$ and the d-spacing to $0.88 \mathrm{~nm}$ as a result of the intercalation 


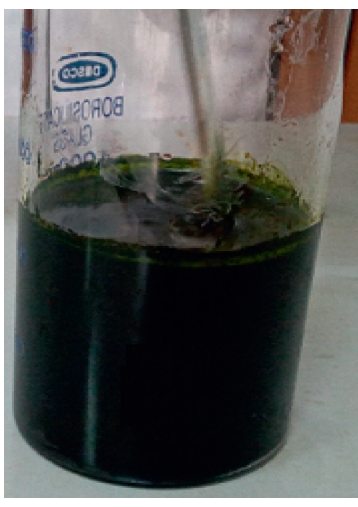

(a)

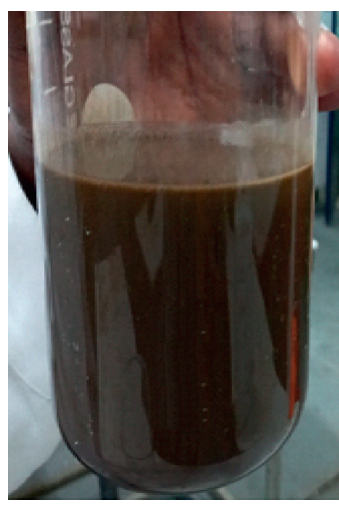

(b)

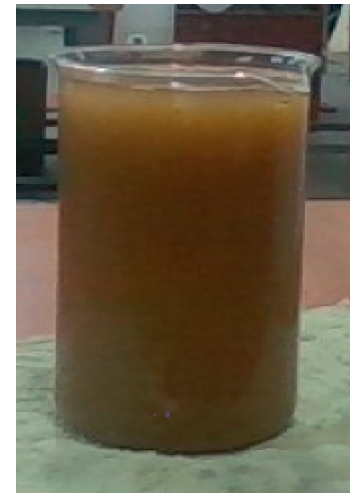

(c)

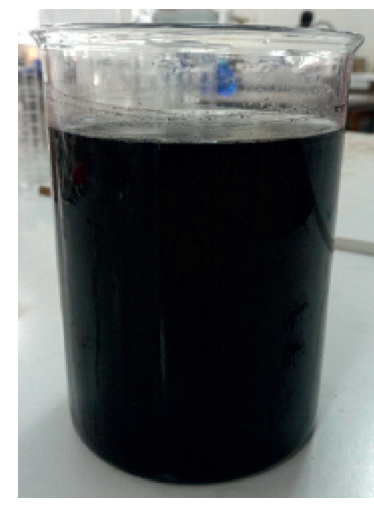

(d)

Figure 1: The color change observed during the stepwise synthesis of reduced graphene oxide.

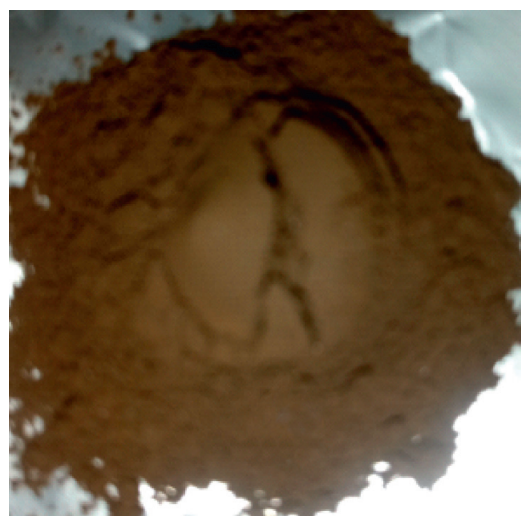

(a)

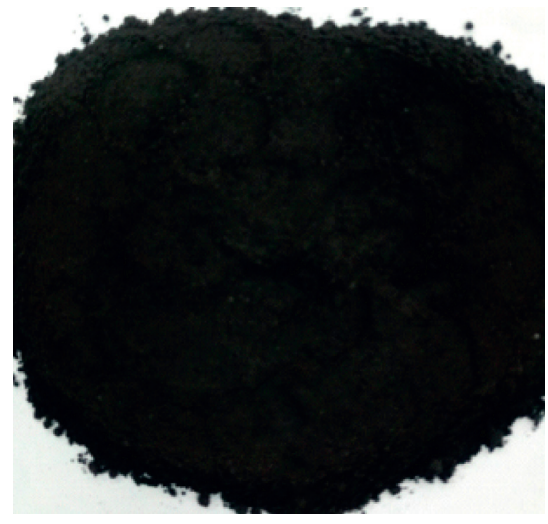

(b)

Figure 2: The powder of GO (a) and rGO (b).

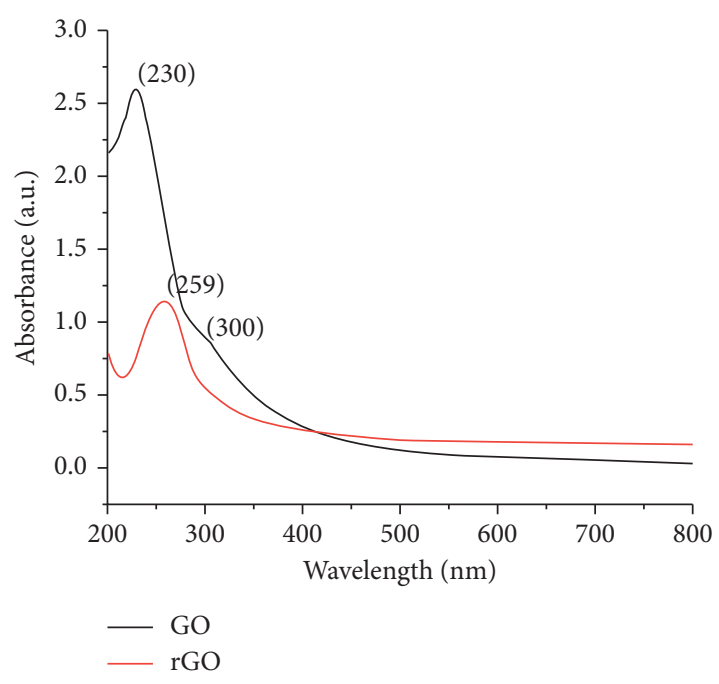

FIGURE 3: UV-Vis spectra record of GO and rGO.

of an oxygen-containing functional group from the oxidizing agent $\mathrm{KMnO}_{4}$ using the intercalating agent $\mathrm{H}_{2} \mathrm{SO}_{4}$. This insertion of an oxygen-containing functional group in

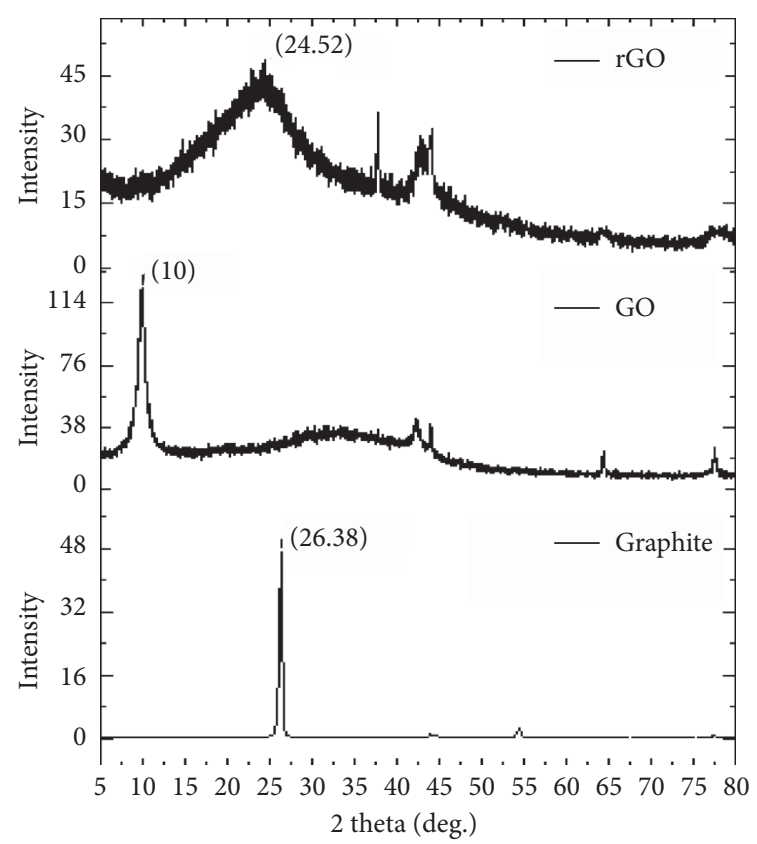

Figure 4: X-ray diffraction patterns of graphite, GO, and rGO. 
the form of hydroxyl and epoxy across the basal plane and carbonyl and carboxyl at the edge of the graphitic structure increases the interlayer distance alarmingly. Thus, the bond between the layers became so weak that they can simply disintegrate to pieces of layers called graphene or rGO by introducing reducing agents.

Furthermore, peaks were observed at around $2 \theta=43^{\circ}$ in GO and rGO which resulted due to slipping out of the alignments of crystal structure from basal planes. With the green reduction using methanol extracts of Vernonia amygdalina (Dhebicha) of GO to produce graphene/(rGO), there was a shift in the d-spacing and $2 \theta$ values to $0.36 \mathrm{~nm}$ and $24.5^{\circ}$, respectively, and this result confirmed the successful reduction of GO to graphene.

3.5. Fourier-Transform Infrared Spectroscopy (FTIR). As shown in Figures 5(a) and 5(b), the structures of the prepared GO and rGO were characterized using FTIR spectroscopy. Figure 5(a) shows a series of different absorption bands or characteristic peaks of GO. The peak at $1735 \mathrm{~cm}^{-1}$ indicates the stretching vibration of carbonyl $(\mathrm{C}=\mathrm{O})$ groups as well as carboxylic acid and the one at $1623 \mathrm{~cm}^{-1}$ attributed to the $(\mathrm{C}=\mathrm{C})$ in the aromatic ring. There was also an absorption peak formed at 1226 and $1051 \mathrm{~cm}^{-1}$ as a result of stretching vibration of epoxide and alkoxy (C-O) group, respectively. The FTIR record of GO displays a broad stretching peak at $3408 \mathrm{~cm}^{-1}$ which was attributed to the $-\mathrm{OH}$ of the intercalated residual water molecules which were almost removed but the remaining small peak is shifted to 3419 in rGO due to reduction.

The weak band displayed at $1376 \mathrm{~cm}^{-1}$ corresponds to the stretching vibration result of $\mathrm{C}-\mathrm{OH}$ of carboxyl originating from carboxylic acids. The bands for the $\mathrm{C}-\mathrm{H}$ symmetric and asymmetric stretching frequencies were observed at 2850 and $2920 \mathrm{~cm}^{-1}$, respectively, in both GO and rGO. The FTIR spectral record of rGO is shown in Figure 5(b). As the oxygencontaining functional groups were removed by the reducing agent, the characteristic absorption peaks of oxide groups decreased significantly, the $\mathrm{C}=\mathrm{O}$ peak at around $1735 \mathrm{~cm}^{-1}$ vanished, and the small shift of bands to the longer wavelength 1738 indicates the successful reduction of GO. The broadband formed at $1638 \mathrm{~cm}^{-1}$ was attributed to the $(C=C)$ stretching of the aromatic ring of the rGO.

3.6. Scanning Electron Microscopy (SEM). SEM is a powerful technique in providing informative data for characterizing microstructure such as corrosion, fracture, grains, and grain boundaries [37]. Figures 6(a)-6(c) show the micrographs for graphite, GO, and rGO at a magnification of $17,000 \mathrm{X}$, $18,000 \mathrm{X}$, and $18,000 \mathrm{X}$, respectively. Figure 6 (a) shows the graphite micrograph which depicts the existence of plateletlike crystalline structure of carbon. The graphite micrograph shown appears to be ordered and compactly stacked with a properly aligned edge because of the existence of Van der Waals forces which bonded their layers together. The SEM morphology of the modified tour method synthesized GO in this work clearly shows a two-dimensional sheet-like structure. As indicated in Figure 6(b), the SEM image of GO showed a smooth, wrinkled structure folded at its surface due to the expansion of the layer because of the insertion of an oxygen-containing functional group during oxidation. The layered flakes observed were the confirmation of complete oxidation of graphite to GO. This resulted in the destroying of the laminated structure in graphite so that the structure of GO is delaminated and disordered. Unlike graphite, GO sheets were thicker at the edge than at the basal planes because of the high combination of an oxygencontaining functional group at the edge. As it can be observed from the SEM image of GO, its layers were independently suspended in the matrix and there is no sign of bending observed.

Figure 6(c) shows the micrograph of rGO obtained by the green reduction of GO using bitter leaf extracts. An improvement in the surface morphology of rGO was observed when compared with the surface morphology of GO because of the removal of wrinkles and folds. After the reduction of GO, different forms of the transparent and thinlayer structure of rGO were obtained. SEM image of rGO demonstrates individual sheet-like structure by means of self-assembly techniques which indicated the successful reduction of GO into rGO by using solvent extracted Vernonia amygdalina.

\subsection{High-Resolution Transmission Electron Microscopy (HR-} TEM). HR-TEM was used to measure the particle size and investigate the morphology of the samples. Figures $7(a)-7(d)$ and Figures $8(a)-8(d)$ show the TEM image recorded for GO and $\mathrm{rGO}$, respectively. These materials were examined under HR-TEM to confirm the formation of GO and rGO and to determine their morphology and size. The EDAX analysis was made to determine the elemental composition of GO and $\mathrm{rGO}$. The result showed that $\mathrm{C}$ and $\mathrm{O}$ were the major components in addition to some impurities such as $\mathrm{S}$ from $\mathrm{H}_{2} \mathrm{SO}_{4}$, Na may be from plant extracts, and Ca may also be from plant extracts, $\mathrm{Cl}$ from $\mathrm{HCl}$ was used for washing purpose, and $\mathrm{K}$ was from the oxidizing agent $\mathrm{KMnO}_{4}$, which must be completely removed during washing. As observed on the graph of EDAX, the percentages of oxygen were greatly reduced in the case of $\mathrm{rGO}$, and this is the indication of the reduction of GO to $\mathrm{rGO}$. As seen from the TEM image of Figures 7(a) and 7(b), the high electron transparent corrugated or wrinkled layered structure of GO was formed. It reveals a mixture of multilayer graphene oxide (dark region) and a few layers of graphene oxide (transparent region). Even if the exact number of $2 \mathrm{D}$ nanosheets cannot be determined, we can observe from the TEM and SEM image that there are no large graphitic aggregates present which indicates the formation of a few layers of graphene. The folded edges observed on the TEM images of rGO were the indicators of the presence of the functional groups. The SEAD pattern displayed the interlayer distance obtained by calculating the distance between two bright spot points around the core white spot matching the values obtained from the XRD analysis.

Figures $8(\mathrm{a})-8(\mathrm{~d})$ represent the $\mathrm{rGO}$ micrograph which displayed plated $\mathrm{rGO}$ from a few layers to a single layer of 


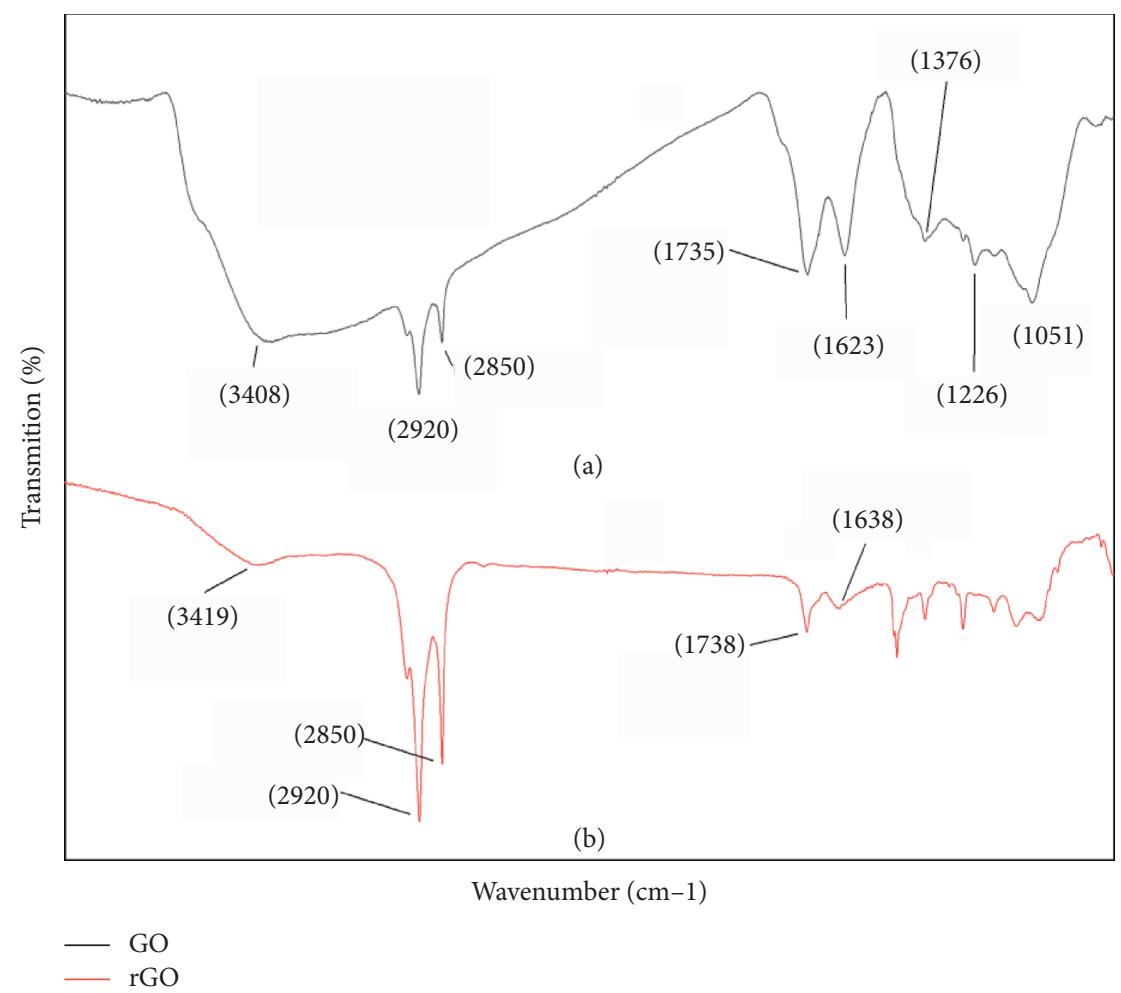

FIGURE 5: FTIR spectra of graphene oxide (a) and reduced graphene oxide (b).

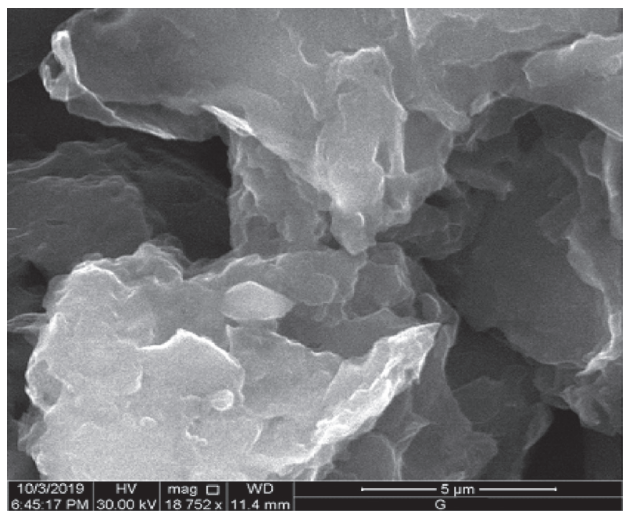

(a)

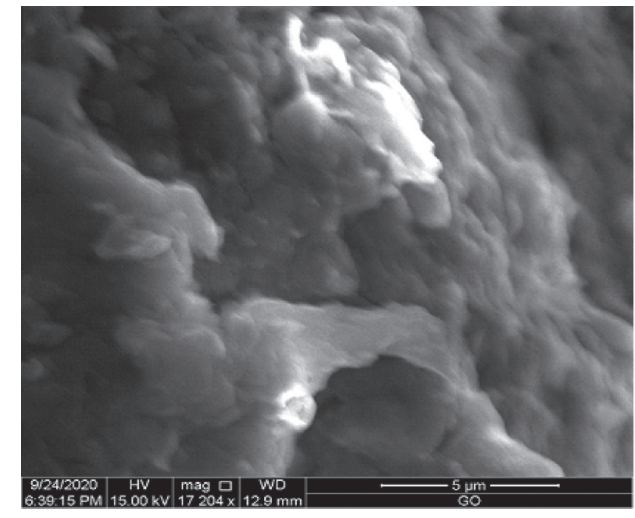

(b)

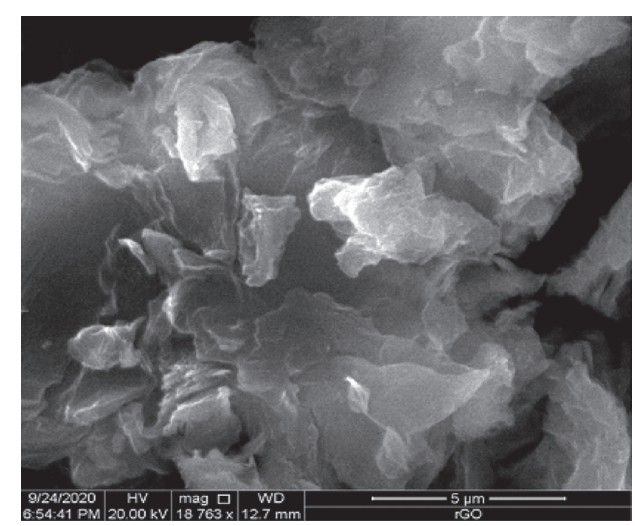

(c)

FIGURE 6: SEM morphology of (a) graphite, (b) GO, and (c) rGO. 


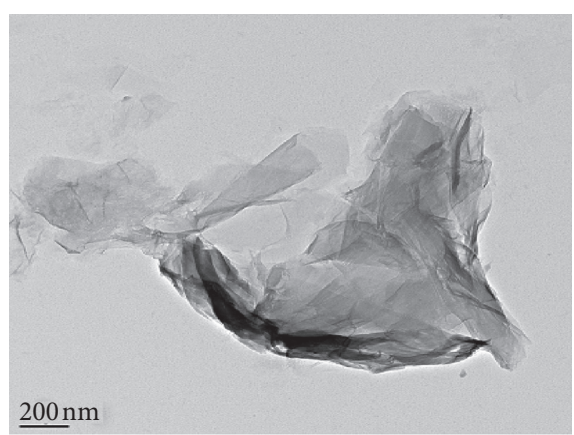

(a)

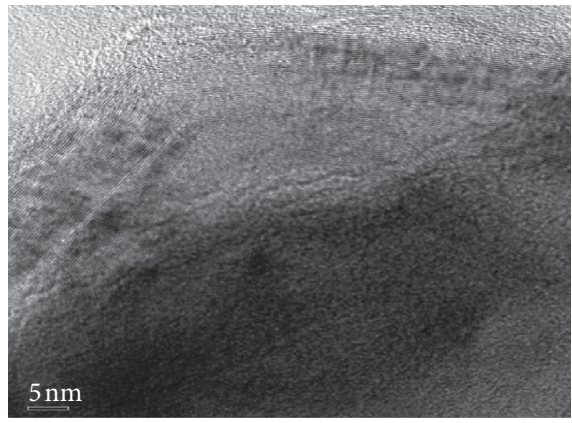

(c)

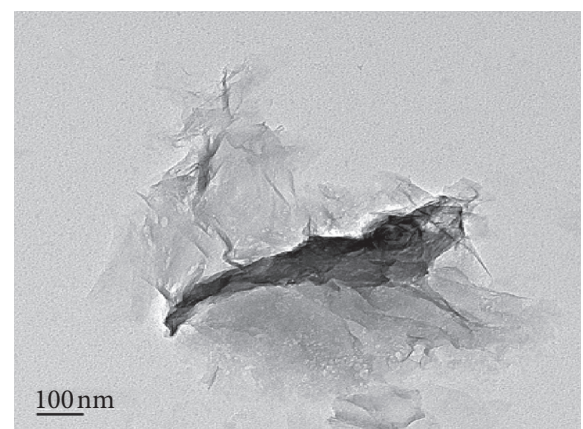

(b)

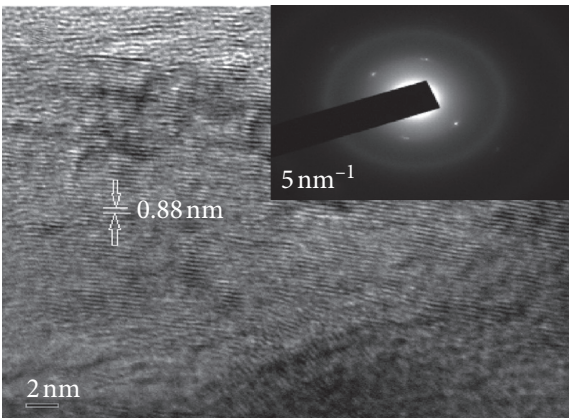

(d)

FIgure 7: (a-d) TEM image of GO along with EDAX analysis, and the inset is SEAD pattern of GO.

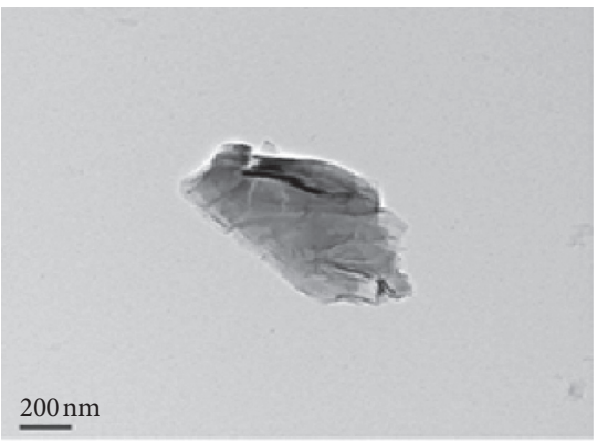

(a)

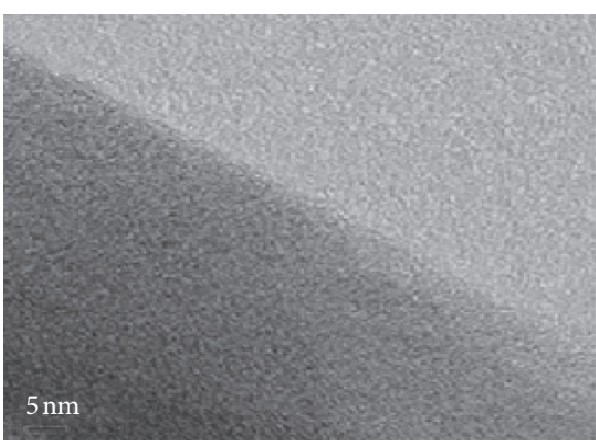

(c)

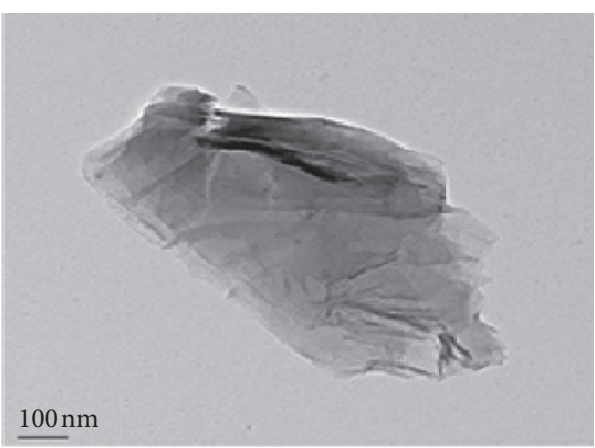

(b)

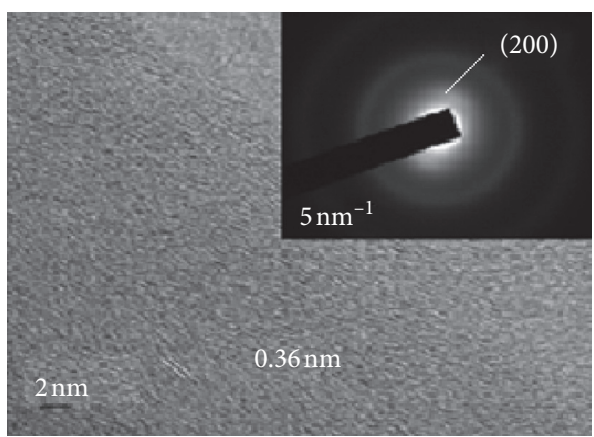

(d)

FIGURE 8: (a-d) TEM image of rGO along with EDAX analysis, and the inset is SEAD pattern of rGO.

graphene. This image depicted that the sample consists of scrolled, wrinkled, and transparent structures which were often the indicators of a few layers of rGO sheets. It can also be clearly seen that the rGO samples are the crippled and wrinkled structure which inhibits the restacking of the graphene sheets together. The TEM micrograph of rGO 


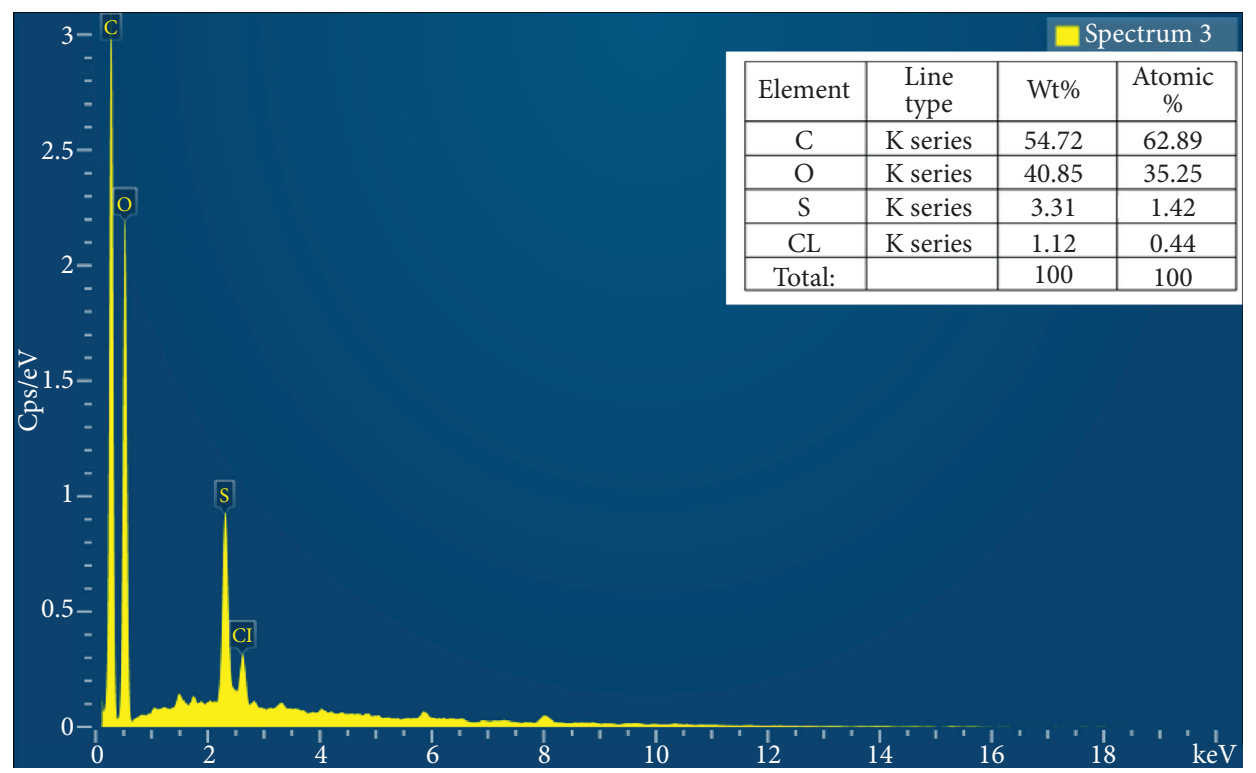

Figure 9: Elemental composition of GO using EDAX.

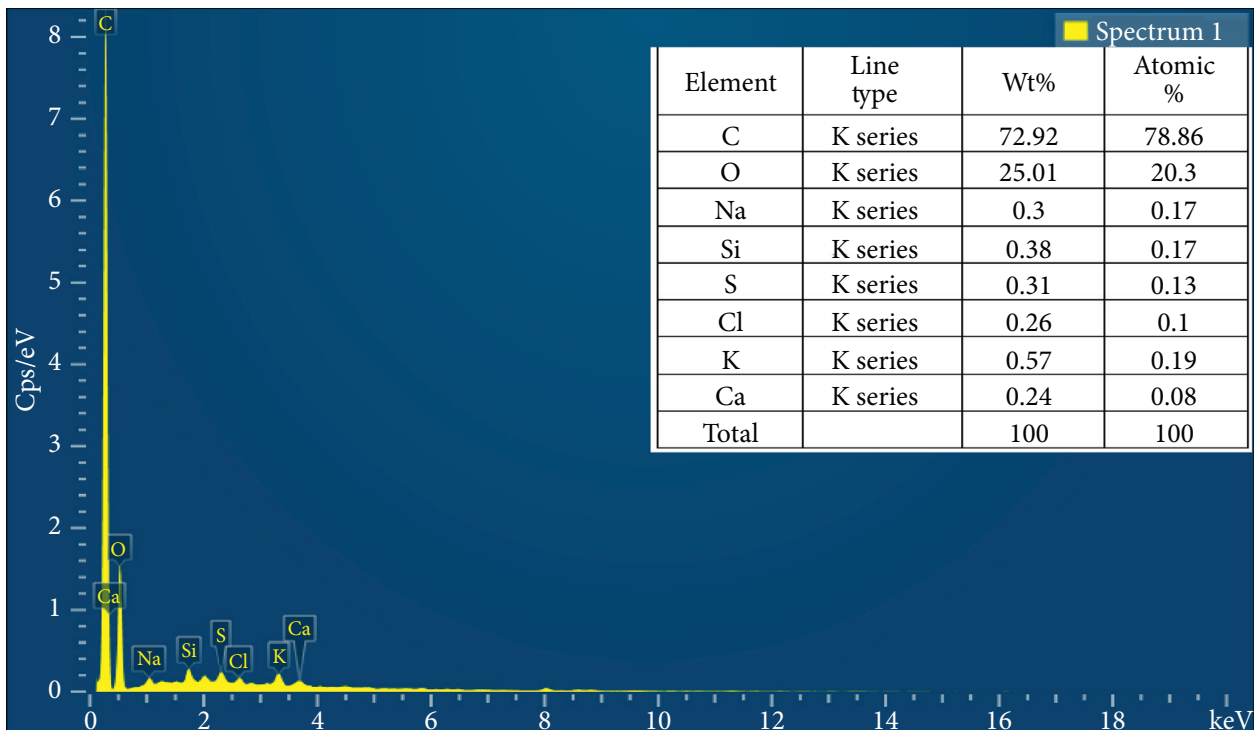

Figure 10: Elemental composition of rGO using EDAX.

revealed that the edge of the sheets appeared to be smooth. The broad XRD peak and the diffused ring indicating the disordered structure and less crystallinity of materials were observed in SAED patterns of rGO.

The energy-dispersive X-ray analysis (EDAX) obtained using K-series shown in Figures 9 and 10 for $\mathrm{GO}$ and $\mathrm{rGO}$, respectively, revealed that the $\mathrm{GO}$ contains $62.89 \%$ of $\mathrm{C}$, $35.25 \%$ of $\mathrm{O}$, and $1.42 \%$ of S. The EDAX analysis suggests that the $\mathrm{rGO}$ sheets contained $78.86 \% \mathrm{C}, 20.30 \%$ of O, $0.17 \%$ of $\mathrm{Si}$, $0.13 \%$ of $\mathrm{S}$, and $0.08 \%$ of $\mathrm{Ca}$ as reported by Rawal et al. [26].

The d-spacing of both GO and rGO was calculated to be $0.88 \mathrm{~nm}$ and $0.36 \mathrm{~nm}$, respectively, from the following equation:

$$
d-\text { spacing }=\frac{2}{\text { distance between the two white spot }} .
$$

These correspond to the (001) and (002) planes of GO and $\mathrm{rGO}$ sheets present at $2 \theta=10^{\circ}$ and $24.5^{\circ}$ of XRD data, respectively, mentioned above.

\section{Conclusion}

A slightly modified tour method was used in this work to synthesis GO which was later used as a precursor for the synthesis of graphene. The nonexplosive and time-saving mixing of two mixtures was done by avoiding the time 
taking slow addition of the highly explosive $\mathrm{KMnO}_{4}$ in the ice bath. The addition of the acids mixture to the mixture of graphite and $\mathrm{KMnO}_{4}$ is much less explosive than the slow addition of $\mathrm{KMnO} 4$ to the other mixture. Easily available, cheap, and green reducing as well as capping agent at the same time with a maximum capacity to reduce $\mathrm{GO}$ to produce graphene having a better solubility in aqueous solvent was discovered from Vernonia amygdalina. The reducing capacity of Vernonia amygdalina was attributed to the existence of a large number of terpenoids and polyphenols in its methanol extracts. This green synthesis method is considered to be the preferred method to manufacture graphene-based materials in a large-scale, ecofriendly, and economical way. The method was tested for its oxygen removing capacity and found to be the efficient and effective reduction method comparable to chemical methods. Different characterization techniques were used to determine the reducing capacity of the plant leaf extracts and confirmed to be the best-reducing compounds for the green synthesis of graphene. Besides, they are capping agents due to their carbonyl and hydroxyl ends in the extracts to stabilize the nanoparticle synthesized.

\section{Data Availability}

The data used in the study are included in the body text of the manuscript.

\section{Conflicts of Interest}

The authors declare that there are no conflicts of interest to disclose.

\section{Acknowledgments}

The authors would like to acknowledge "DST-SAIF Cochin" for the TEM analysis.

\section{References}

[1] Y. Kopelevich and I. A. Luk'yanchuk, "Graphite vs graphene: scientific background," Noble Prize, vol. 50005, p. 10, 2010.

[2] H. Y. Mao, Y. H. Lu, J. D. Lin, S. Zhong, A. T. S. Wee, and W. Chen, "Manipulating the electronic and chemical properties of graphene via molecular functionalization," Progress in Surface Science, vol. 88, no. 2, pp. 132-159, 2013.

[3] F. Torrisi, T. Hasan, W. Wu et al., "Inkjet-printed graphene electronics," ACS Nano, vol. 6, no. 4, pp. 2992-3006, 2012.

[4] S. Ramakrishnan, M. Dhakshnamoorthy, E. J. Jelmy, R. Vasanthakumari, and N. K. Kothurkar, "Synthesis and characterization of graphene oxide-polyimide nanofiber composites," RSC Advances, vol. 4, no. 19, pp. 9743-9749, 2014.

[5] S. Nazarpour, R. Hajian, and M. H. Sabzvari, "A novel nanocomposite electrochemical sensor based on green synthesis of reduced graphene oxide/gold nanoparticles modified screen printed electrode for determination of tryptophan using response surface methodology approach," Microchemical Journal, vol. 154, Article ID 104634, 2020.

[6] S. Ramakrishnan, J. Balamurugan, M. Vinothkannan, A. R. Kim, S. Sengodan, and D. J. Yoo, "Nitrogen-doped graphene encapsulated FeCoMoS nanoparticles as advanced trifunctional catalyst for water splitting devices and zinc-air batteries," Applied Catalysis B: Environmental, vol. 279, Article ID 119381, 2020.

[7] M. Vinothkannan, C. Karthikeyan, G. Gnana Kumar, A. R. Kim, and D. J. Yoo, "One-pot green synthesis of reduced graphene oxide ( $\mathrm{RGO} / \mathrm{Fe} 3 \mathrm{O} 4$ nanocomposites and its catalytic activity toward methylene blue dye degradation," Spectrochimica Acta Part A: Molecular and Biomolecular Spectroscopy, vol. 136, pp. 256-264, 2015.

[8] B. Haghighi and M. A. Tabrizi, "Green-synthesis of reduced graphene oxide nanosheets using rose water and a survey on their characteristics and applications," RSC Advances, vol. 3, no. 32, pp. 13365-13371, 2013.

[9] A. K. Geim, "Nobel lecture: random walk to graphene," Reviews of Modern Physics, vol. 83, no. 3, pp. 851-862, 2011.

[10] M. F. Zainuddin, N. H. Nik Raikhan, N. H. Othman, and W. F. H. Abdullah, "Synthesis of reduced Graphene Oxide (rGO) using different treatments of Graphene Oxide (GO)," IOP Conference Series: Materials Science and Engineering, vol. 358, p. 6, 2018.

[11] B. Deng, Z. Liu, and H. Peng, "Toward mass production of CVD graphene films," Advanced Materials, vol. 31, Article ID 1800996, 2019.

[12] Y. Dong, S. Guo, H. Mao, C. Xu, Y. Xie, and C. Cheng, "The growth of graphene on $\mathrm{Ni}-\mathrm{Cu}$ alloy thin films at a low temperature and its carbon diffusion mechanism," Nanomaterials, vol. 9, pp. 1-11, 2019.

[13] S. Morichi, Y. Komoda, H. Inagaki, H. Itami, and H. Itami, "Examination on the vertical normal strain observed at the ground surface during earthquakes," Doboku Gakkai Ronbunshuu A, vol. 64, no. 2, pp. 452-457, 2008.

[14] F. Perreault, A. Fonseca De Faria, and M. Elimelech, "Environmental applications of graphene-based nanomaterials," Chemical Society Reviews, vol. 44, no. 16, pp. 5861-5896, 2015.

[15] T. Chen and R. Cheung, "Mechanical properties of graphene," Graphene Science Handbook, vol. 90, pp. 3-16, 2016.

[16] M. Hakimi and P. Alimard, "Graphene: synthesis and applications in biotechnology-a review," World Applied Programming, vol. 2, pp. 377-388, 2012.

[17] O. A. Yildirim and T. Ozturk, "Graphene-semiconductor composites as visible light-induced photocatalyst, visible-light photocatal," Carbon-Based Materials, vol. 3, 2018.

[18] P. Kumar, A. Kumar Singh, S. Hussain et al., "Graphene: synthesis, properties and application in transparent electronic devices," Reviews in Advanced Sciences and Engineering, vol. 2, no. 4, pp. 238-258, 2013.

[19] G. Yenny Hernandez, M. Lotya, V. Nicolosi, M. Fiona, D. Blighe Sukanta, and J. N. C. Duesberg, "Liquid phase production of graphene by exfoliation of graphite in surfactant/water solutions Yenny," Journal of the American Chemical Society, vol. 131, no. 10, pp. 3611-3620, 2009.

[20] A. F. Ghanem and M. A. Rehim, "Assisted tip sonication approach for graphene synthesis in aqueous dispersion," Biomedicines, vol. 6, pp. 1-15, 2018.

[21] N. Pachauri, S. Verma, P. Singh, and S. P. Singh, "Few layered graphene oxide thin films: a potential matrix for immunosensors," Integrated Ferroelectrics, vol. 184, no. 1, pp. 85-91, 2017.

[22] S. Joshi, R. Siddiqui, P. Sharma, R. Kumar, G. Verma, and A. Saini, "Green synthesis of peptide functionalized reduced graphene oxide (rGO) nano bioconjugate with enhanced antibacterial activity," Science Reports, vol. 10, pp. 1-11, 2020. 
[23] B. Gürünlü, Ç Taşdelen Yücedağ, and M. R. Bayramoğlu, "Green synthesis of graphene from graphite in molten salt medium," Journal of Nanomaterials, vol. 2020, 2020.

[24] H. A. Hessain and J. J. Hassan, "Green synthesis of reduced graphene oxide using ascorbic acid," Iraqi Journal of Science, vol. 61, pp. 1313-1319, 2020.

[25] G. B. Mahendran, S. J. Ramalingam, J. B. B. Rayappan, S. Kesavan, T. Periathambi, and N. Nesakumar, "Green preparation of reduced graphene oxide by Bougainvillea glabra flower extract and sensing application," Journal of Materials Science: Materials in Electronics, vol. 31, no. 17, pp. 14345-14356, 2020.

[26] N. Singh, P. Siwatch, J. Kaushal, J. Sharma, and S. K. Tripathi, "One-step green synthesis of reduced graphene oxide by electrochemical etching of carbon rods and effect of different bias voltages on the quality," Journal of Solid State Chemistry, vol. 291, Article ID 121537, 2020.

[27] N. Rawal, S. Solanki, and D. Shah, "Green synthesis of reduced graphene oxide with in situ decoration of metal nanoparticles for charge storage application," Materials Today: Proceedings, vol. 21, pp. 2066-2071, 2020.

[28] J. Feng, Y. Ye, M. Xiao, G. Wu, and Y. Ke, "Synthetic routes of the reduced graphene oxide," Chemical Papers, vol. 74, no. 11, pp. 3767-3783, 2020.

[29] Z. Çıplak, B. Getiren, C. Gökalp, A. Yıldız, and N. Yıldız, "Green synthesis of reduced graphene oxide-AgAu bimetallic nanocomposite: catalytic performance," Chemical Engineering Communications, vol. 207, pp. 559-573, 2020.

[30] Y.-Y. Liu, S.-H. Luo, S.-X. Yan, J. Feng, and T.-F. Yi, “Green synthesis of reduced graphene oxide as high-performance electrode materials for supercapacitors," Ionics, vol. 26, no. 1, pp. 415-422, 2020.

[31] S. Manchala, V. S. R. K. Tandava, D. Jampaiah, S. K. Bhargava, and V. Shanker, "Novel and highly efficient strategy for the green synthesis of soluble graphene by aqueous polyphenol extracts of eucalyptus bark and its applications in high-performance supercapacitors," ACS Sustainable Chemistry \& Engineering, vol. 7, no. 13, pp. 11612-11620, 2019.

[32] X. Liu, C. Giordano, and M. Antonietti, "A facile molten-salt route to graphene synthesis," Small, vol. 10, no. 1, pp. 193-200, 2014.

[33] D. C. Marcano, D. V. Kosynkin, J. M. Berlin et al., "Improved synthesis of graphene oxide," ACS Nano, vol. 4, no. 8, pp. 4806-4814, 2010.

[34] A. Jilani, M. H. D. Othman, M. O. Ansari et al., "Graphene and its derivatives: synthesis, modifications, and applications in wastewater treatment," Environmental Chemistry Letters, vol. 16, no. 4, pp. 1301-1323, 2018.

[35] T. Fentaw and D. Worku, "Controlled synthesis, characterization and reduction of graphene oxide: a convenient method for large scale production, Egypt," Journal of Basic \& Applied Sciences, vol. 4, pp. 74-79, 2017.

[36] E. SA Abo El and H. My, "Thermally reduced graphene oxide: synthesis, structural and electrical properties," International Journal of Nanoparticles and Nanotechnology, vol. 3, 2017.

[37] P. Choudhary and O. P. Choudhary, "Scanning electron microscope: advantages and disadvantages in imaging components," International Journal of Current Microbiology and Applied Sciences, vol. 6, no. 5, pp. 1877-1882, 2017. 